\title{
Cobertura vacinal da poliomielite na região Nordeste do Brasil no primeiro ano de pandemia por Covid-19
}

\section{Eucilene Alves Santana ${ }^{1}$, Carmen Lucia Muricy Braz, Tamires Vital ${ }^{3}$ and Helen Gurgel ${ }^{4}$}

\begin{abstract}
Resumo
A Poliomielite foi eliminada no Brasil, desde a década de 90, atribuído ao sucesso das campanhas de vacinação no país, tendo o último caso registrado em 1989. Porém uma baixa cobertura vacinal é um dos fatores de alerta, para a volta da circulação do vírus selvagem no país e das sequelas permanentes provocadas pelo vírus, exigindo assim um monitoramento contínuo no território. Este trabalho teve como objetivo analisar a distribuição espacial da cobertura vacinal (CV) da poliomielite na região Nordeste do Brasil em 2020 quanto a meta de CV $\geq 95 \%$. Os dados públicos do indicador da Taxa de CV foram obtidos do Sistema de Informação do Programa Nacional de Imunizações no site do DATASUS. Os resultados até o momento da extração mostram a Região Nordeste com cobertura de $71,8 \%$ inferior à meta, sendo a menor taxa de CV registrada, no estado do Maranhão $(60,1 \%)$ e a maior, no Ceará (86,9\%). O Estado do Maranhão, também apresentou um maior número de municípios em situação crítica $(23,5 \%)$,) com cobertura vacinał $50 \%$. Os dados espaciais mostram uma tendencia de agrupamento de municípios com alta cobertura vacinal em alguns estados da região, porém não altera a conclusão de que em 2020 a população menor de um ano esteve em situação de vulnerabilidade quanto a reintrodução do vírus selvagem na maior parte dos municípios dos estados dessa região e nos demais estados brasileiros.
\end{abstract}

\section{Palavras-chave}

Análise de espacial, indicador, paralisia flácida 


\section{Introdução}

A Poliomielite, doença altamente contagiosa, caracterizada por um quadro clínico de paralisia flácida com início súbito, tem como agente etiológico o poliovírus, pertencente ao gênero Enterovírus, família Picornaviridae, composto por três sorotipos 1, 2 e 3, tendo como reservatório, o homem. A transmissão ocorre principalmente, por contato direto, pessoa a pessoa, pelas vias fecal-oral, ou por objetos, alimentos e água contaminada por fezes de doentes ou de portadores e ainda, pela via oral-oral, por gotículas de secreções da orofaringe ao falar, tossir ou espirrar (Saúde \& Bolso, 2010). Não há cura para a doença, apenas a prevenção por vacinas

Essa doença encontra-se eliminada no Brasil, desde a década de 90, tendo seu último caso em 1989, como resultado do sucesso das campanhas de vacinação no país. A circulação do vírus selvagem foi eliminada, porém fatores como, más condições habitacionais, higiene pessoal precária e o elevado número de crianças em uma mesma habitação podem favorecer a transmissão do poliovírus (Saúde \& Bolso, 2010).

A baixa Cobertura Vacinal (CV), também é um fator de alerta, na volta da circulação do vírus selvagem no país e/ou surgimento de Poliovírus Derivado Vacinal (PVDV). Segundo a Sociedade Brasileira de Medicina Tropical, em um informe sobre o perigo da reintrodução da poliomielite, pesquisadores chamam a atenção para a baixa cobertura vacinal, a qual favorece ao vírus vivo enfraquecido das vacinas orais, a continuar a se multiplicar no intestino humano (raro, mas possível), mudar geneticamente até o ponto de recuperar a capacidade de causar paralisia e se espalhar naqueles que não foram vacinados. Os surtos registrados em países da África do Sul, por exemplo tiveram como origem o poliovírus derivado da vacina, diferente dos dois únicos países Paquistão e Afeganistão que ainda possuem em seu território, cepas remanescentes do poliovírus selvagem (SBMT, 2020).

O Brasil em 2015, publicou o "Plano de Erradicação da Poliomielite: Estratégia no Brasil" com o objetivo geral de atingir a meta global de erradicação da poliomielite, contendo seis objetivos específicos: criar o Comitê Nacional de Certificação da Erradicação da Poliomielite; fortalecer a Vigilância Epidemiológica das Paralisias Flácidas Agudas (VE PFA); substituir a vacina oral atenuada trivalente pela bivalente; realizar a contenção laboratorial do poliovírus; implantar gradualmente a Vigilância Ambiental do poliovirus; e mobilizar os profissionais de saúde e sociedade civil de forma eficaz para a divulgação da nova estratégia (Saúde \& Saúde, 2015).

\footnotetext{
${ }^{1}$ LAGAS, Universidade de Brasília

${ }^{3}$ Departamento de Imunização e Doenças Transmissíveis-Secretaria de Vigilância em Saúde, Ministério da Saúde

${ }^{4}$ Departamento de Geografia, Universidade de Brasíla, Brasília, Distrito Federal, Brasil
}

Corresponding author:

Eucilene Alves Santana, Universidade de Brasília

Email: eucilene.alvessantana@gmail.com 
Além da possível interação e mudança genética, pesquisas epidemiológicas sugerem que vacinas vivas atenuadas como a vacina oral contra poliomielite (VOP), podem resultar na indução de efeitos não específicos no sistema imunológico (Blok, Arts, Crevel, Benn, \& Netea, 2015). mas também pode proteger contra outros tipos de vírus (Higgins et al., 2016; Aaby e Benn 2017; Upfill-Brown et. al., 2017) (Higgins et al., 2016; Upfill-Brown et al., 2017).

O Brasil, no entanto, introduziu a vacina inativada contra poliomielite (VIP) para todos os bebês que iniciam sua série de vacinação primária, desde 2012, com o objetivo de prevenção de casos raros de poliomielite paralítica, associada à vacina, garantindo o acesso equitativo à VIP e preparando-se para a futura cessação da VOP após a erradicação global, com o devido monitoramento contínuo para o alcance da alta cobertura vacinal (Domingues et al., 2016).

Porém, os estados e os municípios brasileiros apresentam características particulares que vão desde culturas, barreiras geográficas, econômicas, ambientais e sociais distintas em único país, e ouvi-los é o primeiro passo para o sucesso na implementação e operacionalização de ferramentas de monitoramento e avaliação em relação ao Plano de Erradicação da Poliomielite: Estratégia no Brasil.

Nesse contexto, este trabalho de natureza exploratório, com foco no território, teve como objetivo a análise do indicador da cobertura vacinal da poliomielite, no período de 2016 a 2020, na região Nordeste, para verificar o alcance da meta de cobertura vacinal ( $\geq 95 \%)$ nesta região.

\section{Metodologia}

Utilizou-se como fonte de dados o Sistema de Informação do Programa Nacional de Imunizações (SIPNI) do Ministério da Saúde, com consulta dos dados públicos, disponíveis no link (http://sipni.datas us.gov.br/si-pni-web/faces/inicio.jsf),selecionando a opção Tabnet (cobertura vacinal) e as variáveis de interesse: Município- linha; Ano- coluna; Coberturas vacinais- conteúdo; Anos 2016 a 2020 -Período; Poliomielite - opção do Imuno.

O indicador Cobertura Vacinal (CV) de Poliomielite é calculado pela equipe do Ministério da Saúde, a partir do número de doses aplicadas do imunobiológico para poliomielite registrado no SI-PNI dividido pela população menores de um ano do banco de dados do Sistema de Nascidos Vivos (SINASC) multiplicado por 100 . Foi utilizada a meta de $95 \%$, preconizada pela OMS e adotada pelo PNI, para se considerar baixa cobertura $(<95 \%)$ ou alta $(\geq 95 \%)$.

As possíveis inconsistências e/ou missing foram verificadas por meio do software Epi InfoTM do Center of Disease Control and Prevention (CDC) e do editor de planilhas Microsoft, Excel, os quais analisaram as frequências das coberturas para cada município. Os mapas com os resultados da cobertura vacinal de poliomielite foram elaborados com RStudio, software livre de ambiente de desenvolvimento integrado para R e com os pacotes: geobr, ggplot, ggspatial, ggrepe.

\section{Resultados}

A cobertura vacinal de poliomielite no Brasil em 2020, até a data de 23 de novembro de 2021, estava em $76,0 \%$, menor do que a cobertura vacinal alcançada nos anos de 2016 a 2019. Segundo dados de nascidos vivos, disponibilizados pelo Ministério da Saúde em 2020, nasceram 1.722.907 crianças (Ministério da Saúde, 2020), destas possivelmente 416.943 crianças não foram vacinadas para poliomielite no país.

Entre as 27 Unidades da Federação (Tabela 1), predomina-se uma CV de Poliomielite entre 51\% e $94 \%$ ( cor amarelo) menor que a meta pactuada ( $\geq 95 \%$ ). Os estados que alcançaram a meta (cor verde) 
de vacinação foram: Rondônia de 2016 a 2019, Ceará de 2016 a 2018; Alagoas, Minas Gerais e Mato Grosso do Sul em 2018 e Santa Catarina em 2017. Porém, em 2020 nenhum dos estados alcançaram a meta de CV de Poliomielite. Dentre os estados chama atenção, o Amapá com a menor cobertura vacinal (41,9\%) em 2020 (cor vermelha), enquanto Santa Catarina apresentou a melhor CV (88,3\%) no mesmo ano.

A região Nordeste alcançou uma cobertura de 71,8\%, em 2020, a menor registrada no período analisado (2016-2019) e a máxima registrada foi de 90,0\% em 2019. Entre os estados, a variação da cobertura, em 2020, foi de 60,1\% a mínima, no estado do Maranhão e de 86,8\% a máxima para o Estado do Ceará, a mediana entre os estados, foi de 71,5\%, abaixo da mediana brasileira.

A tabela 2 apresenta a síntese da situação dos municípios por estados da região Nordeste. O Estado com maior número de municípios é a Bahia (417) e, o menor, Sergipe com 75 municípios. Os estados da região Nordeste apresentaram municípios com cobertura vacinal menor que $50 \%$, considerado de alto risco para a recirculação do vírus selvagem. O Estado com maior número de municípios nessa situação é o Maranhão: $23,5 \%$ dos seus municípios estão com cobertura inferior ou igual a $50 \%$, e o menor percentual de municípios nesta situação foi registrado nos estados do Ceará (6,0\%) e Paraíba (7,2\%).

Observou-se, também, que em todos os estados, têm-se municípios com registro de cobertura vacinal superior a $100 \%$. O estado de Alagoas teve menor percentual de municípios (10,8\%) com cobertura superior a 100\%, enquanto o estado da Paraíba o maior percentual de municípios nesta situação (34,5\%).

Em 2018 houve um avanço quanto a distribuição de municípios no estado e Alagoas, com CV igual ou maior que 95\%, porém em 2019 é possível ver uma redução territorial dessa proteção, e em 2020 poucos municípios com cobertura superior a 95\%, restrito a 37 municípios (20\%), alguns destes de área fronteiriça com outros estados (Figura 1)

No estado da Bahia a maior concentração de municípios, com cobertura igual ou maior que 95\%, estão localizados próximos aos limites dos estados de Minas Gerais, Tocantins, Goiás e para municípios limítrofes, totalizando 88 (21\%) dos 417 municípios do estado em 2020. Nos anos anteriores a maior abrangência territorial foram nos anos de 2018 e 2019 (Figura 2).

Um dos estados com maior distribuição de municípios com cobertura vacinal igual ou maior que $95 \%$, em 2020, tanto no interior do estado quanto na sua região litorânea e em municípios limítrofes, foi o Ceará, dos 184 municípios, 59 (32\%) estão com cobertura $\geq 95 \%$. Nos municípios deste estado é nítido o efeito da pandemia quando comparado com os demais anos, com maior parte do seu território com meta alcançada de CV. Chama atenção municípios que por dois anos consecutivos deixaram de informar (NAem cinza) a cobertura vacinal no sistema oficial do país (Figura 3).

Enquanto o estado do Maranhão com 217 municípios, apenas 36 (16\%) dos seus municípios alcançaram cobertura vacinal igual e/ou superior a 95\% em 2020. Apesar dos demais anos também não apresentarem um avanço, as melhores CV se repetem em municípios de fronteiras ou municípios limítrofes'conforme a resolução Normativa $n^{\circ}$ 259, 17 de junho de 2011-ANS. Na figura 4, em 2020 esses municípios estão distribuídos de forma aleatória no estado ou são municípios limítrofes.

No estado da Paraíba, 223 (43\%) municípios que alcançaram a meta $(95 \%)$ em 2020, e estão distribuídos em todo o estado, no entanto, chama atenção o agrupamento de municípios limítrofes com boa cobertura vacinal. Este estado, apresentou maior percentual de municípios com boa cobertura vacinal 
Tabela 1. Cobertura Vacinal de Poliomielite nas UF do Brasil de 2016- 2020

\begin{tabular}{|c|c|c|c|c|c|}
\hline Unidade da Federação & 2016 & 2017 & 2018 & 2019 & 2020 \\
\hline Rondônia & 105,4 & 108,2 & 101,9 & 98,3 & 82,0 \\
\hline Acre & 71,3 & 74,0 & 78,3 & 81,7 & 62,8 \\
\hline Amazonas & 76,2 & 76,4 & 79,3 & 83,3 & 67,4 \\
\hline Roraima & 88,5 & 90,5 & 79,8 & 79,8 & 72,9 \\
\hline Pará & 63,2 & 67,6 & 69,1 & 72,7 & 59,2 \\
\hline Amapá & 47,6 & 63,2 & 68,7 & 73,0 & 41,9 \\
\hline Tocantins & 84,8 & 86,1 & 91,7 & 88,2 & 83,8 \\
\hline Maranhão & 69,4 & 74,3 & 80,6 & 75,7 & 60,1 \\
\hline Piauí & 70,7 & 78,1 & 83,7 & 81,9 & 71,5 \\
\hline Ceará & 107,9 & 97,3 & 111,1 & 93,5 & 86,9 \\
\hline Rio Grande do Norte & 70,3 & 69,5 & 90,3 & 80,7 & 69,6 \\
\hline Paraíba & 85,5 & 82,3 & 92,3 & 92,6 & 72,6 \\
\hline Pernambuco & 90,4 & 84,7 & 94,7 & 85,6 & 71,7 \\
\hline Alagoas & 80,1 & 83,9 & 96,1 & 87,9 & 72,1 \\
\hline Sergipe & 78,3 & 79,0 & 89,7 & 80,9 & 70,6 \\
\hline Bahia & 70,7 & 78,3 & 78,3 & 74,8 & 69,7 \\
\hline Minas Gerais & 88,3 & 87,1 & 97,8 & 88,5 & 85,9 \\
\hline Espírito Santo & 89,3 & 83,2 & 91,0 & 86,7 & 81,6 \\
\hline Rio de Janeiro & 89,9 & 88,8 & 87,5 & 73,6 & 55,3 \\
\hline São Paulo & 83,8 & 87,7 & 92,6 & 86,6 & 82,0 \\
\hline Paraná & 87,5 & 90,4 & 90,9 & 89,7 & 86,1 \\
\hline Santa Catarina & 92,1 & 95,1 & 94,6 & 93,7 & 88,3 \\
\hline Rio Grande do Sul & 84,5 & 85,7 & 85,7 & 85,1 & 84,8 \\
\hline Mato Grosso do Sul & 93,8 & 91,5 & 96,0 & 94,4 & 81,9 \\
\hline Mato Grosso & 90,6 & 84,1 & 90,3 & 85,8 & 80,8 \\
\hline Goiás & 82,1 & 81,6 & 85,5 & 81,5 & 77,8 \\
\hline Distrito Federal & 136,8 & 84,4 & 86,0 & 84,3 & 81,5 \\
\hline Brasil & 84,4 & 84,7 & 89,5 & 84,2 & 76,0 \\
\hline
\end{tabular}


Tabela 2. Análise descritiva das informações da cobertura vacinal dos 1.794 municípios por estado, da região Nordeste do Brasil de 2020.

\begin{tabular}{lccc|cccc}
\hline \multicolumn{1}{c}{ Estado } & \multicolumn{3}{c}{ Número de municípios } & \multicolumn{5}{c}{$\begin{array}{c}\text { Dados de cobertura vacinal } \\
\text { n (\%) }\end{array}$} \\
& \multicolumn{2}{c}{ Por } & Com & Sem & $50 \%$ & $51 \%$ a $94 \%$ & $95 \%$ a \\
& Estado & dados & dados & & $100 \%$ & $>100 \%$ \\
\hline Maranhão & 217 & 217 & 0 & $51(23,5)$ & $132(60,8)$ & $11(5,1)$ & $23(9,8)$ \\
Piauí & 224 & 224 & 0 & $20(8,9)$ & $130(58,0)$ & $12(5,4)$ & $62(27,7)$ \\
Ceará & 184 & 184 & 0 & $11(6,0)$ & $114(62,0)$ & $16(8,7)$ & $43(23,4)$ \\
Rio Grande do & 167 & 166 & 1 & $14(8,4)$ & $105(63,2)$ & $2(1,2)$ & $45(27,1)$ \\
Norte & 223 & 223 & 0 & $16(7,2)$ & $111(49,8)$ & $19(8,5)$ & $77(34,5)$ \\
Paraíba & 185 & 185 & 0 & $20(10,8)$ & $112(60,5)$ & $16(8,6)$ & $37(20,0)$ \\
Pernambuco & 102 & 102 & 0 & $11(10,8)$ & $71(69,6)$ & $9(8,8)$ & $11(10,8)$ \\
Alagoas & 75 & 75 & 0 & $8(10,7)$ & $45(60)$ & $6(8,0)$ & $16(21,3)$ \\
Sergipe & 417 & 417 & 0 & $35(8,4)$ & $294(70,5)$ & $26(6,2)$ & $62(14,9)$ \\
Bahia & & & & & & & \\
\hline
\end{tabular}

em 2020 quando comparado aos demais estados na região nordeste do país. Porém os anos mais críticos foram 2020 e 2017 (Figura 5).

Em 2020, dos 185 municípios do estado de Pernambuco, 53\% (29\%) alcançaram a meta de cobertura vacinal para poliomielite( ( $\geq 5 \%$ ). Estes são visualizados no tom de azul-escuro, formando pequenos agrupamentos no estado, por serem municípios limítrofes em sua maioria e/ou municípios próximos dos estados do Ceará e Paraíba. No entanto, este foi um dos anos com menor distribuição de municípios com alcance da meta, assim como no ano de 2017 (Figura 6).

No estado do Piauí com 224 municípios, 74(33\%) destes em 2020, apresentaram cobertura $¥ 95 \%$ ), formando agrupamentos de municípios limítrofes em todo o estado. O melhor desempenho de vacinação contra a Poliomielite ocorreu em 2017 quando comparado com os demais anos. Porém, este é um dos estados quem em 2016 teve município que não informou (NA- em cinza) a CV no sistema oficial do país (Figura 7).

O estado do Rio Grande do Norte em 2020 foi o único estado com município sem informação sobre a cobertura vacinal de Poliomielite (NA - cor cinza), situação registrada também em 2016 para outro município do estado. Dos 167 municípios, 47(28\%) atingiram a meta da CV $\$ 95 \%$ ) em sua população de menores de um ano. As melhores coberturas foram observadas em municípios limítrofes no estado em 2020 e a melhor cobertura no território foi registrada em 2018 (Figura 8). 
General Maynard. Não diferente de alguns estados, o ano de 2018 foi o que apresentou maior distribuição de municípios com alcance da meta vacinal (Figura 9).

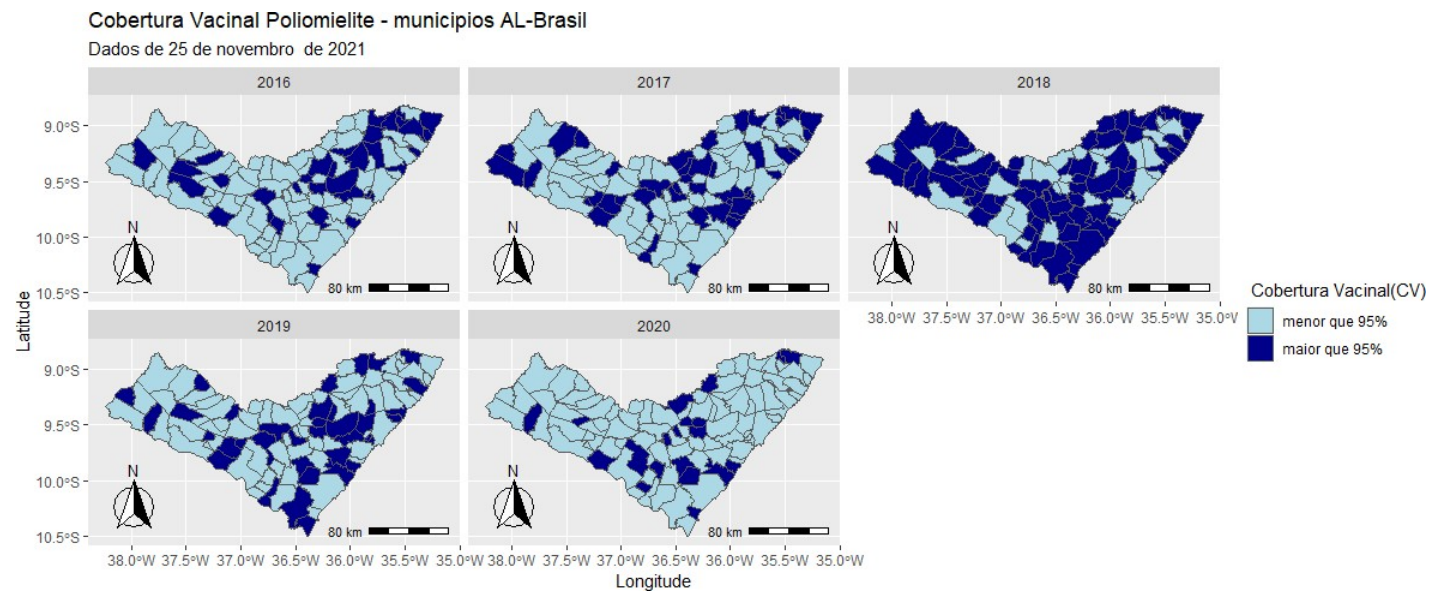

Figure 1. Cobertura Vacinal de Poliomielite dos municípios estado de Alagoas de 2016 a 2020. Elaboração própria. Fonte: SI-PNI.

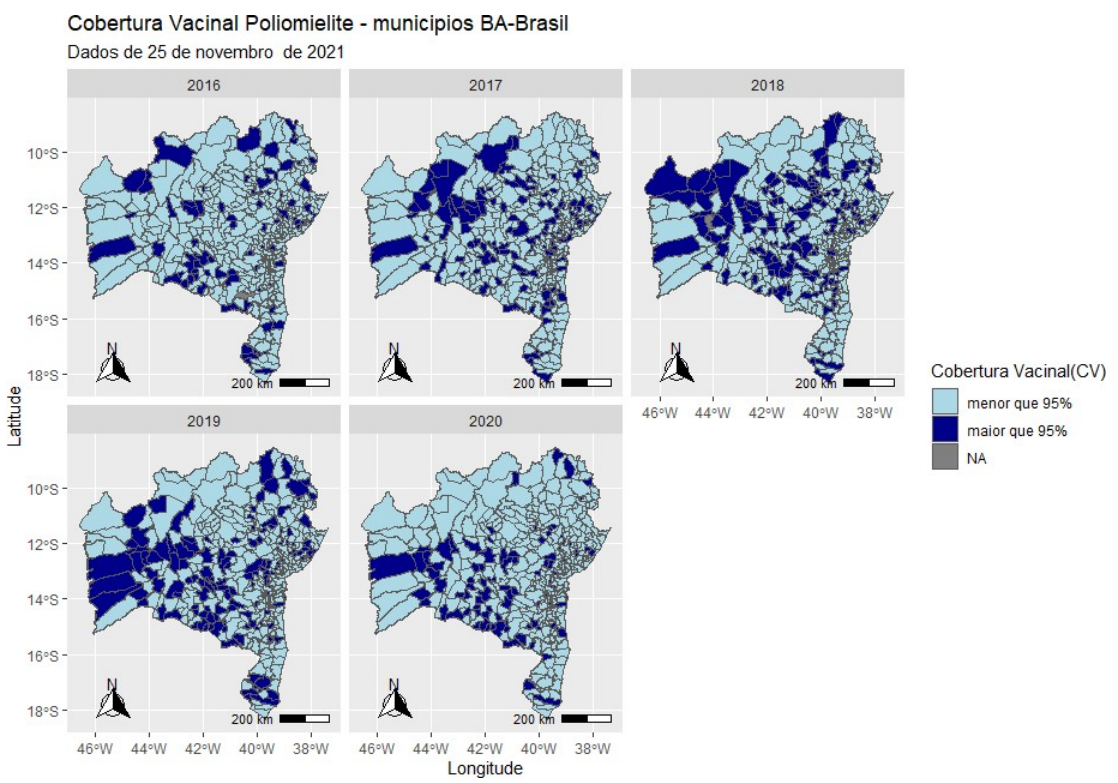

Figure 2. Cobertura Vacinal de Poliomielite dos municípios daBahia de 2016 a 2020. Elaboração própria. Fonte: SI-PNI. 


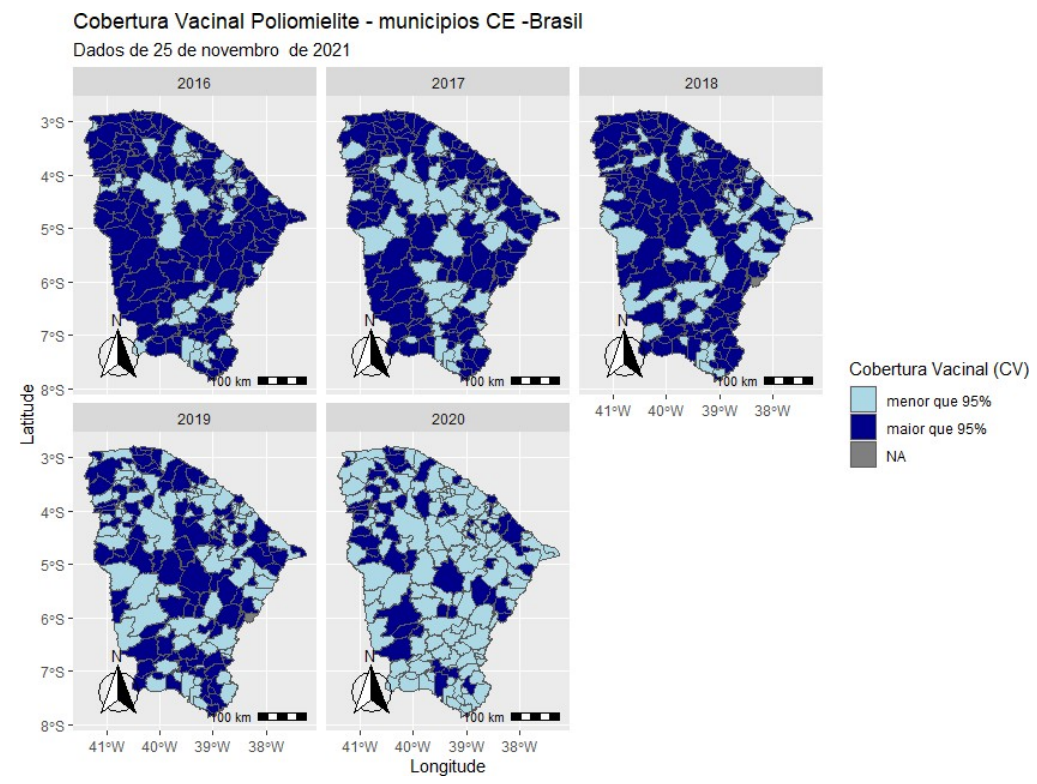

Figure 3. Cobertura Vacinal de Poliomielite dosmunicípios do Ceará de 2016 a 2020. Elaboração própria. Fonte: SI-PNI.

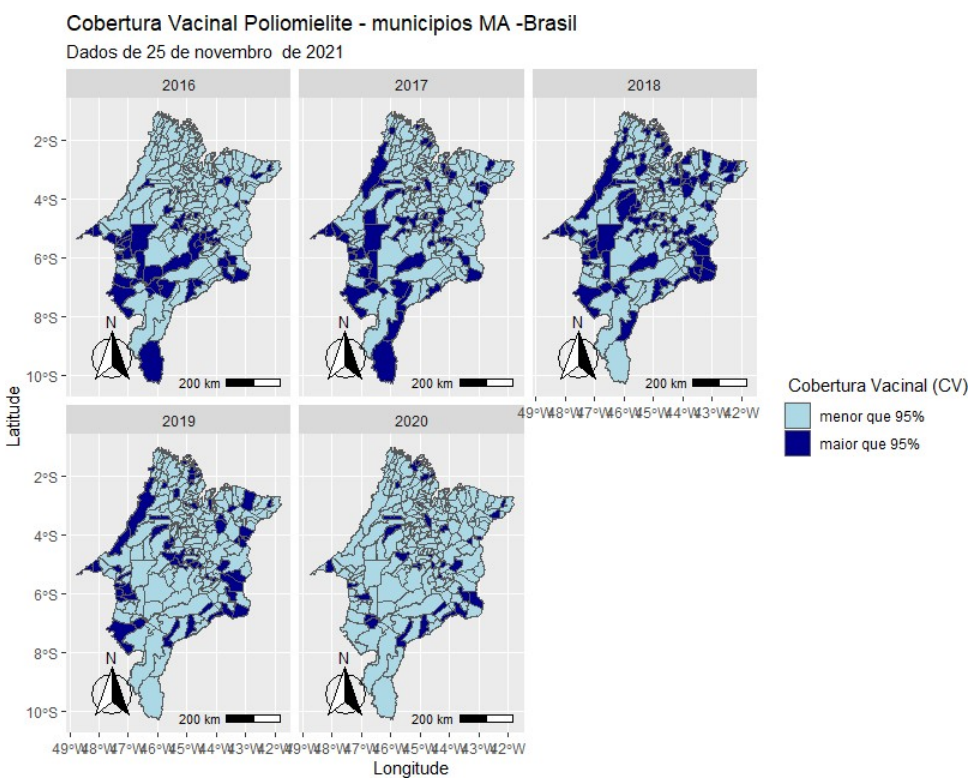

Figure 4. Cobertura Vacinal de Poliomielite dos municípios doMaranhão de 2016 a 2020. Elaboração própria. Fonte: SI-PNI. 


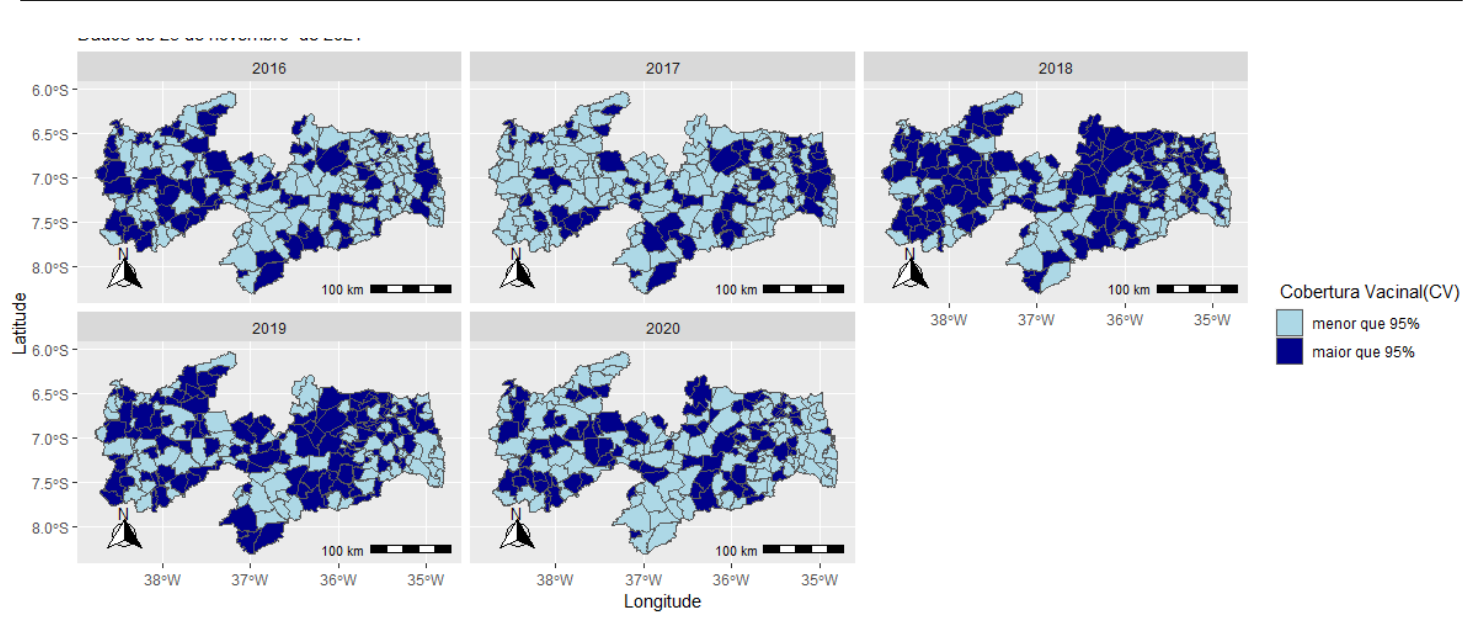

Figura 5. Cobertura Vacinal de Poliomielite dos municípios do estado da Paraíba de 2016 a 2020. Elaboração própria. Fonte: SI-PNI.

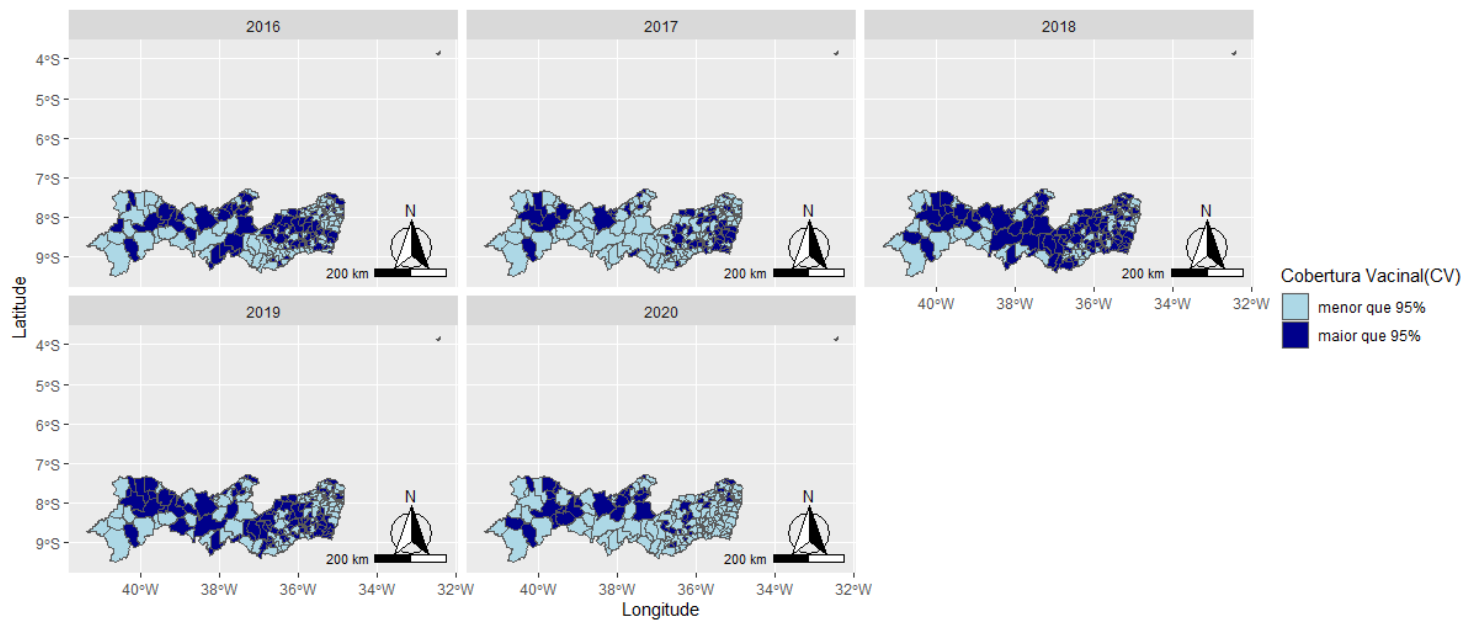

Figura 6. Cobertura Vacinal de Poliomielite dos municípios de Pernambuco de 2016 a 2020. Elaboração própria. Fonte: SI-PNI. 


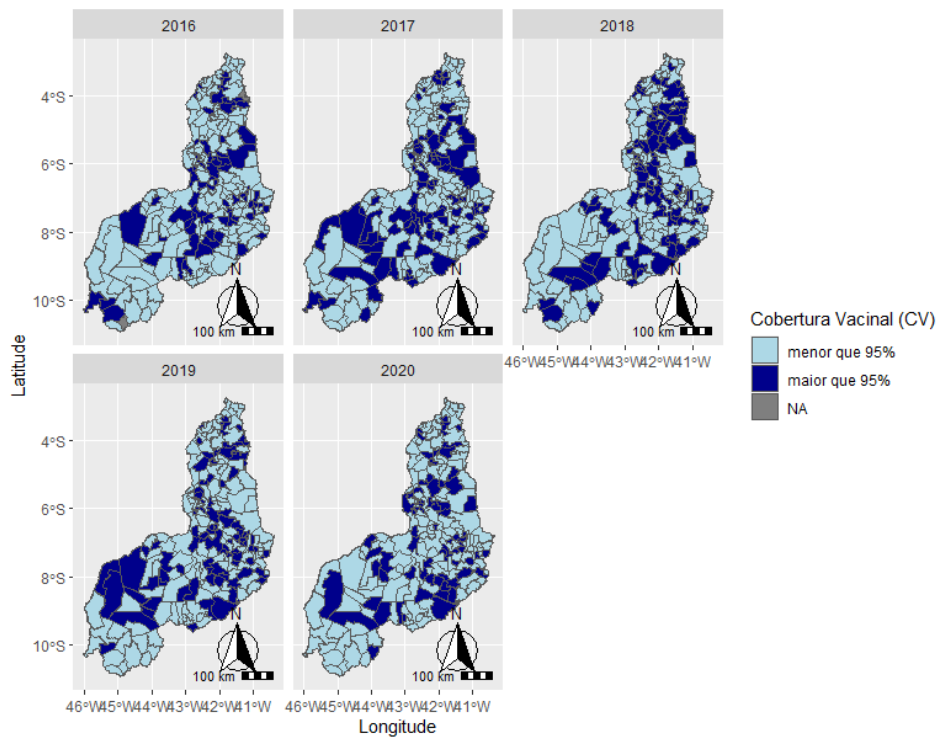

Figura 7. Cobertura Vacinal de Poliomielite dos municípios do estado do Piauí de 2016 a 2020. Elaboração própria. Fonte: SI-PNI.
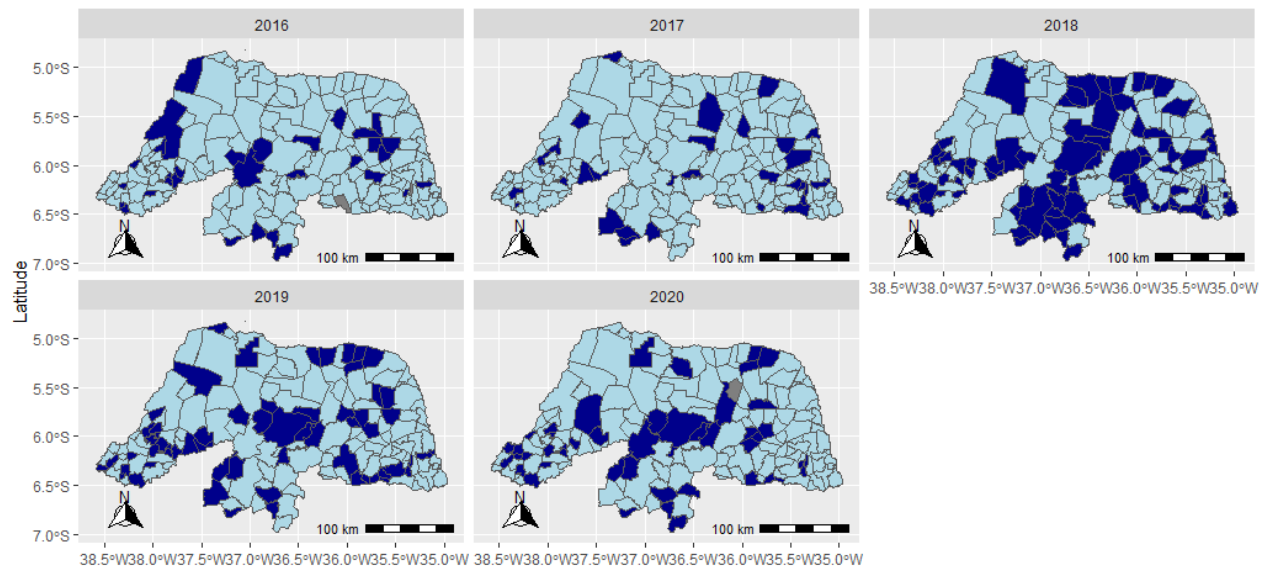

Cobertura Vacinal(CV)

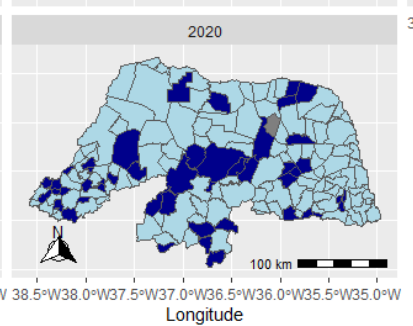

menor que $95 \%$

maior que $95 \%$ NA

Figura 8. Cobertura Vacinal de Poliomielite dos municípios do estado do Rio Grande do Norte de 2016 a 2020. Elaboração própria. Fonte: SI-PNI. 


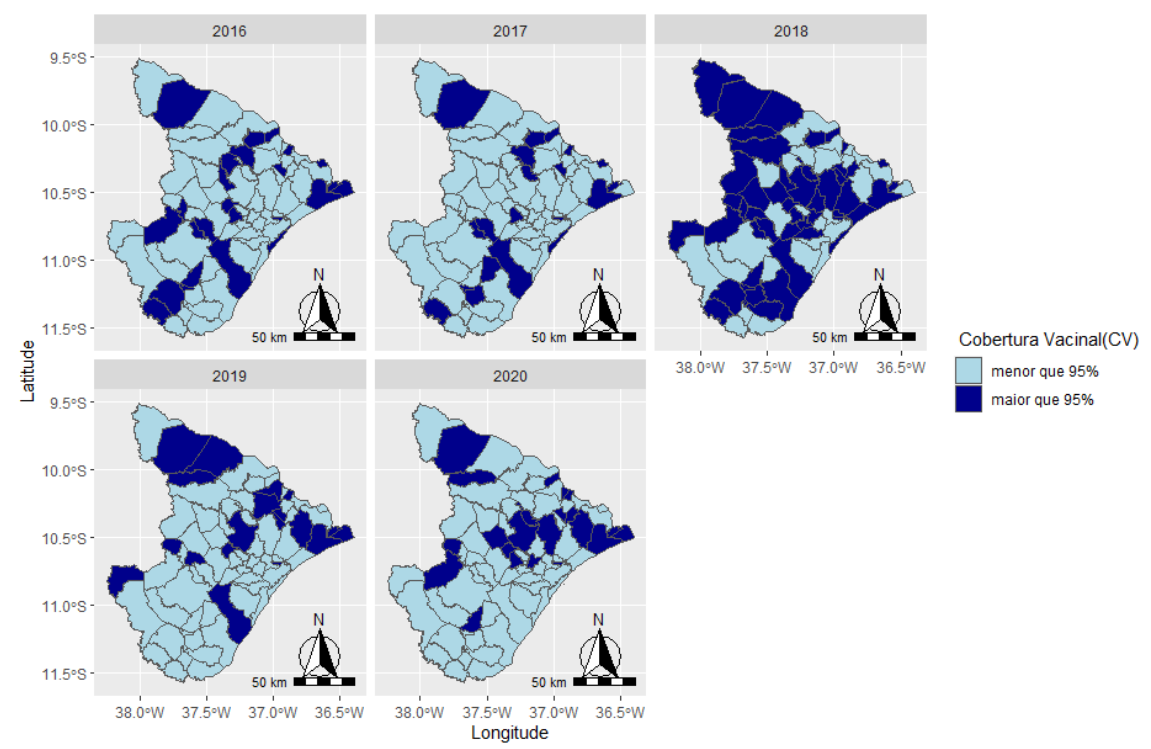

Figura 9. Cobertura Vacinal de Poliomielite dos municípios do estado de Sergipe de 2016 a 2020. Elaboração própria. Fonte: SI-PNI.

\section{Considerações Finais}

Os resultados de 2020, para a região Nordeste, mostram a acentuada queda da cobertura vacinal da poliomielite, que pode ter sido agravada em consequência da pandemia que o país e o mundo vivenciam desde fevereiro de 2020. Todas as ações de saúde estão voltadas para conter o avanço do vírus SARSCoV-2 e ao tratamento oportuno dos casos graves, e, consequentemente, com a interrupção ou diminuição das ações e serviços de saúde, desenvolvidos pela atenção básica/primária.

Os resultados óbitos para o ano de 2020, evidenciam a necessidade do fortalecimento das ações e das campanhas referentes à poliomielite nesta região. Ações ou campanhas com a oferta de todas as vacinas do calendário do SUS, sugerem uma boa estratégia para o alcance de grande parte da população alvo. Segundo gestores de saúde do estado da Paraíba, o "Dia D" realizado em outubro de 2020, favoreceu o aumento da cobertura de Poliomielite nesse ano no estado (Governo do Estado da Paraíba, 2020). O estado do Ceará na Região Nordeste também foi um dos estados que aderiram ao "Dia D" Campanha de vacinação que ocorreu, também em outubro de 2020, conforme notícias na mídia digital. No entanto, não foram localizadas informações sobre o "Dia D” nos demais estados da região nordeste dopaís.

Em 2020, em torno de 214.490 (28,2\%) dos nascidos vivos na região Nordeste não receberam a dose para a prevenção da poliomielite. Sendo necessário verificar se o não acesso teve como determinante 
o ano pandêmico que se iniciou com a circulação do SARS-CoV2, ou se outros fatores como, a falta de infraestrutura nos municípios, localidades remotas, nascidos vivos de imigrantes ou a resistência à vacinação contribuíram com a baixa cobertura na região.

A baixa cobertura vacinal observada na região, pode favorecer o ressurgimento da doença, pois ainda, há um alto quantitativo de municípios nos estados dessa região, com cobertura abaixo de $50 \%$. A realização de investigação e diagnóstico dessa baixa cobertura nestes municípios são necessários com o objetivo de verificar quais os fatores e as causas destes municípios estarem no grupo de risco para o ressurgimento da doença.

Segundo Fugitaet al. (2018), no Brasil várias razões podem estar contribuindo para a baixa cobertura, a exemplo de desinformação sobre a importância das vacinas, do calendário administrativo e da hesitação vacinal. Um problema que vem ocorrendo com nações desenvolvidas, e que pode também estar ocorrendo no Brasil, com um grande alcance por meio das redes e mídias sociais maior do movimento antivacina nas em relação à não importância das vacinas preconizadas na saúde pública brasileira.

Os municípios com cobertura vacinal acima de $100 \%$, identificados na região nordeste sugerem o possível uso do somatório de doses aplicadas por imunobiológico, ao invés da contagem do número de crianças vacinadas, ou ainda imprecisões do registro de dose de vacinas aplicadas, principalmente quando das realizações de campanhas de vacinação.

Os dados espaciais sugerem que em alguns estados tem cluster de municípios com altas coberturas enquanto outros estados, os municípios estão dispersos, e que tanto no interior quanto as capitais estão com problemas em alcançar a cobertura vacinal para poliomielite. Autores como Fugita et al. (2018), destacam a necessidade de medidas urgentes para incluir o aumento das taxas de cobertura vacinal e ainda, o uso da sorologia de doenças preveníveis por vacinas, como ferramenta de triagem para identificar pacientes que precisam de vacinação.

Para alcançar os objetivos especificados no Plano desde 2015, diante do cenário do indicador de cobertura vacinal, anos com pandemia de SARS-Cov-2, um diagnóstico prévio deve ser realizado com a participação dos gestores municipais e/ou estaduais para a identificação de experiências bem-sucedidas e/ou das dificuldades encontradas para o alcance da meta de cobertura pactuada. Uma das formas de permitir que todos os 5.570 municípios brasileiros possam oferecer a sua população o acesso à vacina em tempo oportuno e proporcionar a barreira necessária para a não recirculação do vírus selvagem no Brasil.

Destaca, ainda que a elaboração de um plano de mitigação, uma matriz de risco e o plano de

monitoramento sem a participação dos gestores dos municípios, local onde as ações de saúde acontecem, poderão apresentar dificuldades quanto a implementação e a operação dessas ferramentas pelos municípios. Pois, segundo Minayo (1995), o Brasil é formando por vários "Brasis". E essas particularidades devem ser consideradas na elaboração de um plano de mitigações e de uma matriz de risco em relação a doença.

Com a permanência da Pandemia e as interrupções dos serviços da Atenção Primária, uma análise situacional será necessária para avaliar as interrupções ocorridas em 2020 no programa de imunização. Tendo impacto direto no acesso aos serviços, devido ao distanciamento físico e reduções de transporte, preocupações de cuidadores e profissionais de saúde sobre a exposição a COVID-19 (Shet, Carr, Danovaro-Holliday et al., 2021). 
Para minimizar este cenário, os estados e municípios em especial devem buscar, estratégias de recuperação para incluir atividades mais imediatas, como atividades de vacinação em massa e comunicação direcionada às pessoas doses da vacina faltantes, desenvolver uma estrutura de vacinação de recuperação dentro da vacinação de rotina, melhorando o rastreamento de inadimplentes, treinando profissionais de saúde para incorporar estratégias de recuperação no programa de imunização, triagem de crianças para o status de vacinação em qualquer encontro de serviço de saúde ou na entrada na escola, e expansão da elegibilidade baseada na idade para vacinações para garantir que crianças mais velhas não vacinadas recebam vacinas perdidas (Muhoza, Danovaro-Holliday, Diallo, etal., 2021).

Os dados permitem concluir que as taxas de cobertura de imunizações para Poliomielite no Brasil e na região Nordeste do país diminuíram em meio a Pandemia de Covid-19, aflorando o risco da reintrodução da doença na região. A CV está abaixo também da taxa global estimada para 2020 (83\%), o que traz implicações na prática de saúde pública e ainda que há alto número de municípios utilizando sistemas que ainda notificam doses aplicadas ao invés do registro individual dos vacinados.

A visualização espacial da CV dos municípios enfatiza a necessidade de novos trabalhos para entender os cluster formados, se estes estão relacionados com a o processo de regionalização do Sistema Único de Saúde, ou se se estão influenciados pela cultura política burocrática, deficiência do planejamento, fragmentação do sistema, ou mesmo pelo movimento antivacina que vem avançando no Brasil.

\section{Agradecimentos}

Ao Grupo de Técnico da Poliomielite do Ministério da Saúde pelas orientações quanto a forma de obtenção de dado e detalhamento das variáveis de análise e ao apoio do Laboratório de Geografia Ambiente e Saúde da Universidade de Brasília (LAGAS/UnB) e do LMI-Sentinela (UnB/FIOCRUZ/IRD).

\section{Referências}

AABY, P.; BENN, C.S., Beneficial nonspecific effects of oral polio vaccine (OPV): implications for the cessation of OPV? Clinical Infectious Diseases, US, 1, 65, 420-421, aug. 2017. Acesso em 22 de abril de 2021. doi: 10.1093/cid/cix340

BLOK, B. A.; ARTS, R. J.; CREVEL, V. C.; BENN. C. S.; NETEA, M. G.; Trained innate immunity as underlying mechanism for the long-term, nonspecific effects of vaccines. Journal Leukocyte Biology, 98, 3, 347-356, sep. 2015. Acesso em 23 de abril de 2021.doi: 10.1189/jlb.5RI0315-096R

CONSELHO NACIONAL DE SECRETARIAS MUNICIPAIS DE SAÚDE (CONASEMS). Nota: Plano de erradicação da Poliomielite no Brasil. Disponível em: https://www.conasems.org.br/nota-plano-deerradicacao-da-poliomelite-no-brasil. Divulgado em 14 de janeiro de 2016. Acesso em 22 de abril de 2021. 
DOMINGUES, C. M.; DE FÁTIMA PEREIRA, S.; CUNHA MARREIROS, A. C.; MENEZES, N.; FLANNERY, B. Introduction of sequential inactivated polio vaccine-oral polio vaccine schedule for routine infant immunization in Brazil's National Immunization Program. J Infect Dis, 1, 210, 143-151, Suppl 1(0 1), nov. 2014. doi: 10.1093/infdis/jit588

FUJITA, D.; SALVADOR, F. S.; NALI, L. H. daS.; LUNA, E. J. deA. Decreasing vaccine coverage rates lead to increased vulnerability to the importation of vaccine-preventable diseases in Brazil.Journa lof Travel Medicine,25, 1, 2018, Acesso em 22 de abril de 2021. doi.org/10.1093/jtm/tay100

GOVERNO DO ESTADO DA PARAÍBA. Paraíba tem melhor cobertura da campanha de vacinação contra poliomielite do Nordeste. Disponível em: https://paraiba.pb.gov.br/noticias/paraiba-tem-melhorcobertura-da-campanha-de-vacinacao-contra-poliomielite-do-nordeste. Publicado em 19 de outubro de 2020. Acesso em 22 de abril de 2021.

HARZING, A. W. 2007.Publish or Perish. Disponível em: https://harzing.com/resources/publish-orperish.

HIGGINS, J. P.; SOARES-WEISER, K.; LOPEZ-LOPEZ, J. A.; KAKOUROU, A.; CHAPLIN, K.; CHRISTENSEN, H.; MARTIN, N. K.; STERNE, J. A. C.; REINGOLD, A. L. Association of BCG, DTP, and measles containing vaccines with childhood mortality: systematic review. BMJ, 355, 5170, 2016. doi:10.1136/bmj. i5170.

MINAYO, M.C. S.; Os Muitos Brasis: Saúde e população na década de 80, São Paulo. Rio de Janeiro: Hucitec, 1995.

MINISTÉRIO DA SAÚDE. Secretaria de Vigilância em Saúde. Análise de Saúde e Vigilância de Doenças Não transmissíveis. Painel de Monitoramento de Nascidos Vivos. Disponível em: http://svs.aids.gov.br/dantps/centrais-de-conteudos/paineis-de-monitoramento/natalidade/nascidos-

vivos/.Acesso em 22 de abril de 2021.

MINISTÉRIO DA SAÚDE. Guia de Bolso. Doenças infecciosas e parasitárias. Brasil. Ministério da Saúde. Secretaria de Vigilância em Saúde. Departamento de Vigilância Epidemiológica. Doenças infecciosas e Parasitárias: Guia de bolso/Ministério da Saúde, Secretaria de Vigilância em Saúde, Departamento de Vigilância Epidemiológica. 8. ed. rev. - Brasília: Ministério da Saúde, 2010. p. 328333. Il. - (Série B. Textos Básicos de Saúde). Disponível em: https://bvsms.saude.gov.br/bvs/publicacoes/doencas_infecciosas_parasitaria_guia_bolso.pdf. Acesso em 22 de abril de 2021. 
MINISTÉRIO DA SAÚDE. Secretaria de Vigilância em Saúde. Coordenação Geral de Programa de Imunizações. Plano de erradicação da Poliomielite: Estratégias no Brasil. Amo 2015. Disponível em: https://portalarquivos.saude.gov.br/images/pdf/2016/abril/07/Plano-pólio--Brasil--07-04-2016.pdf.

Acesso em 22 de abril de 2021.

MUHOZA P, DANOVARO-HOLLIDAY MC, DIALLO MS, et al. Routine Vaccination Coverage Worldwide, 2020. MMWR Morb Mortal Wkly Rep 2021;70:1495-1500. DOI: http://dx.doi.org/10.15585/mmwr.mm7043a1

RIO GRANDE DO SUL. Secretaria da Saúde, Departamento de Ações em Saúde. Coordenação Estadual da Atenção Básica. Boletim Informativo $n^{\circ} 10$ de novembro de 2020: Programa Previne Brasil: monitoramento do indicador de desempenho 5: Cobertura vacinal de Poliomielite inativada e de Pentavalente. $10^{\mathrm{a}}$ edição. Governo do Estado do Rio Grande do Sul. Disponível em: https://atencaobasicaadmin.rs.gov.br/upload/arquivos/202012/08093414-10-boletim-vacina.pdf. Acesso em 20 de abril de 2021.

SHET A, CARR K, DANOVARO-HOLLIDAY MC, et al. Impacto da pandemia SARS-CoV-2 nos serviços de vacinação de rotina: evidências de interrupção e recuperação em 169 países e territórios. SSRN [Pré-impressão publicada online em 26 de maio de 2021]. https://papers.ssrn.com/sol3/papers.cfm?abstract_id=3850009

SOCIEDADE BRASILEIRA DE MEDICINA TROPICAL (SBMT). Perigo da reintrodução da poliomielite. Disponível em: https:/www.sbmt.org.br/portal/perigo-de-reintroducao-da-poliomielite/. Publicado em 08 de janeiro de 2020. Acesso em 22 de abril de 2021.

UPFILL-BROWN, A.; TANIUCHI, M.; PLATTS-MILLS, J. A.; KIRKPATRICK, B.; BURGESS, S. L.; OBERSTE, M. S.; WELDON, W.; HOUPT, E.; HAQUE, R.; ZAMAN, K.; PETRI, W. A. Jr. Nonspecific effects of oral polio vaccine on diarrheal burden and etiology among Bangladeshi infants. Clinical Infectious Diseases, Oxford, 65, 414-419, 2017. Acesso em 22 de abril de 2021.doi:10.1093/cid/cix354 